\title{
Theoretical Aspects of Nanoparticle Electric Propulsion
}

\author{
Thomas M. Liu ${ }^{*}$, Michael Keidar ${ }^{\dagger}$, Louis D. Musinski ${ }^{\ddagger}$, Alec D. Gallimore ${ }^{\S}$, and Brian E. Gilchrist ${ }^{* *}$ \\ University of Michigan, Ann Arbor, MI, 48109
}

The nanoparticle field extraction thruster (nanoFET), using charged nanoparticles to generate propulsive thrust, is currently under development at the University of Michigan. This paper discusses the theoretical aspects of nanoFET operation, including particle charging, transport, and extraction from the liquid reservoir. Considerations regarding the liquid, such as Taylor cone formation and colloid generation as well as space charge limits associated with a viscous medium, are also discussed. The paper concludes with a discussion of the relationship between particle scaling and thruster performance.

\section{Nomenclature}

\begin{tabular}{|c|c|}
\hline A & $=$ cylindrical particle aspect ratio \\
\hline$D$ & $=$ drag force \\
\hline$d$ & $=$ electrode gap \\
\hline$d_{l}$ & $=$ liquid layer thickness \\
\hline$E$ & $=$ electric field \\
\hline$E_{l}$ & $=$ liquid electric field \\
\hline$E_{0}$ & $=$ vacuum electric field \\
\hline$E_{0, \text { min }}$ & $=$ minimum vacuum electric field to cause liquid surface instability \\
\hline$F_{\text {buoyant }}$ & $=$ buoyant force \\
\hline$F_{\text {inertial }}$ & $=$ inertial force \\
\hline$F_{\text {surface }}$ & $=$ surface tension force \\
\hline$g_{0}$ & $=$ gravitational acceleration at Earth's surface \\
\hline$I_{\mathrm{sp}}$ & $=$ specific impulse \\
\hline$j$ & $=$ current density \\
\hline$j_{\mathrm{CL}}$ & $=$ Child-Langmuir current density \\
\hline$K$ & $=$ "added mass" coefficient \\
\hline$k$ & $=$ wave number \\
\hline l & $=$ cylindrical particle length \\
\hline$m_{l}$ & $=$ liquid mass displaced by particle \\
\hline$m_{p}$ & $=$ particle mass \\
\hline$n$ & $=$ particle number density \\
\hline$P$ & $=$ jet power \\
\hline$q$ & $=$ particle charge \\
\hline$q_{0}$ & $=$ initial particle charge from charging electrode \\
\hline$q_{0, \mathrm{cy}-\mathrm{h}}$ & $=$ initial charge on horizontal cylindrical particle \\
\hline$q_{0, \mathrm{cy}-\mathrm{v}}$ & $=$ initial charge on vertical cylindrical particle \\
\hline$q_{0, \mathrm{sp}}$ & $=$ initial charge on spherical particle \\
\hline$r$ & $=$ particle radius \\
\hline$T$ & $=$ thrust \\
\hline$t$ & $=$ time \\
\hline
\end{tabular}

\footnotetext{
* Graduate Student, Aerospace Engineering, liutm@umich.edu, AIAA Student Member

${ }^{\dagger}$ Assistant Research Professor, Aerospace Engineering, keidar@umich.edu, AIAA Senior Member

${ }^{\ddagger}$ Graduate Student, Electrical Engineering, louisdm@umich.edu, AIAA Student Member

$\S$ Arthur F. Thurnau Professor, Aerospace Engineering \& Applied Physics, rasta@umich.edu, AIAA Associate Fellow

** Professor, Electrical Engineering \& Space Systems, gilchrst@umich.edu, AIAA Associate Fellow
} 


$\begin{array}{ll}t_{\mathrm{ex}} & =\text { particle extraction time } \\ V & =\text { bias voltage across electrodes } \\ v & =\text { particle velocity } \\ v^{*} & =\text { characteristic particle velocity } \\ V_{l} & =\text { voltage drop in liquid layer } \\ V_{O} & =\text { accelerating potential } \\ W & =\text { particle weight } \\ Z & =\text { axial coordinate } \\ \alpha & =\text { drag factor } \\ \beta & =\text { field enhancement factor } \\ \gamma & =\text { surface tension coefficient } \\ \varepsilon_{l} & =\text { permittivity of liquid } \\ \varepsilon_{0} & =\text { permittivity of free space } \\ \mu_{l} & =\text { dynamic viscosity coefficient of liquid } \\ \rho_{l} & =\text { liquid mass density } \\ \sigma_{l} & =\text { liquid electrical conductivity } \\ \tau & =\text { charge loss time constant } \\ \varphi & =\text { electric potential } \\ \omega & =\text { wave frequency }\end{array}$

\section{Introduction}

$\mathrm{R}$ ECENTLY, a new concept was proposed to use highly scalable MEMS/NEMS (micro/nano-electromechanical systems) technologies applied to nanoparticle electric propulsion. ${ }^{1}$ Using nanoparticles permits the tuning of the specific impulse $\left(I_{\mathrm{sp}}\right)$ and thrust over a very broad range. When high $I_{\mathrm{sp}}$ is required, small nanoparticles can be emitted; for lower $I_{\mathrm{sp}}$, larger nanoparticles can be used for higher thrust capability. $I_{\mathrm{sp}}$ and thrust range can be controlled by the size, shape, and density of the particles as well as the acceleration potential.

The use of nanoparticles can be contrasted with the formation of small droplets in colloid thrusters. With the right emission current and temperature, charge extraction in colloid thrusters can produce instabilities that result in the formation of charged microscopic droplets (colloids). ${ }^{2}$ While these droplets could in principle be used to accomplish the same propulsive goal as nanoparticles, droplet sizes are difficult to control, and a size spreading is expected that reduces thrust controllability. Using nanoparticles under conditions that avoid the generation of colloids permits the tuning of propulsion performance via the charge-to-mass ratio.

This nanoparticle electric propulsion concept, under development at the University of Michigan, is named the nanoparticle field extraction thruster (nanoFET), schematically shown operating in a dielectric liquid in Figure 1. The nanoFET system uses conductive nanoparticles of well characterized dimensions and charge states. These nanoparticles are suspended in a low vapor pressure liquid, which is circulated and transported to extraction zones through microfluidic channels. At the extraction zones, the particles are charged via contact with an electrode that is immersed in the dielectric liquid. When particles acquire sufficient charge, they are lifted off the electrode by an imposed electric field provided by the biased MEMS gate structures. Particles are transported to the liquid surface by the electrostatic force and must then overcome the surface tension force to be extracted from the liquid. Following extraction, the particles are accelerated by the imposed electric field to provide thrust.

Significant operational and performance advantages may be achieved with nanoFET over other electric propulsion technologies. These advantages include:

- Highly integrated system: The use of MEMS technology enables a "flat panel" thruster design that incorporates power processing as well as nanoparticle manufacture, storage, feed, extraction, and acceleration. Such compact design simplifies propulsion system integration and lowers thruster specific mass. Because different 
regions of nanoFET can emit particles of opposite polarity, neutralizer requirements are simplified as nanoFET is a self-neutralizing thruster.

- Geometrically scalable: A "flat panel" design enables a single thruster type, which can be straightforwardly scaled, to span a broad power range. This "plug-and-play" technology decouples thruster design from spacecraft design and provides mission planners with greater flexibility.

- Operational lifetime not driven by primary life-limiting factors of other electric propulsion systems: Charging of the nanoparticles is accomplished without ionization, meaning greater reliability and the absence of cathodes, which are one of the principal lifetime limiters of current electric propulsion systems. Propellant charging, as opposed to propellant ionization, also accounts for part of the efficiency gains nanoFET affords.

- Better controllability: Charge-to-mass ratio is much better regulated for nanoparticles than for colloids, resulting in better controllability for nanoFET versus colloidal thrusters.

- Higher thrust density: Whereas state-of-the-art ion thrusters operate substantially below the space charge limit to ensure proper ion optics operation, nanoFET should operate much closer to the space charge limit since the charges will be contained in a fewer number of particles. Thus, thruster specific mass would be reduced.

- Enormous specific impulse range at high thrust efficiency: The ability to tune propulsion characteristics via different nanoparticle dimensions and charge states should permit thrust efficiencies over $90 \%$ for a specific impulse range of 100 to 10,000 seconds, as seen in Figure 2. Such high efficiencies would result in thrust-topower ratios, especially at low specific impulse, that are greater than state-of-the-art ion and Hall thrusters. This capability would be enhanced by the ability to manufacture tailor-made nanoparticles in situ. Consequently, nanoFET could operate at high specific impulse in cruise mode and yet switch to a high thrust mode when needed. This flexibility provides a wider margin for mission designers to accommodate offnominal mission scenarios as well as dynamic retasking of space assets to take advantage of in-flight opportunities.

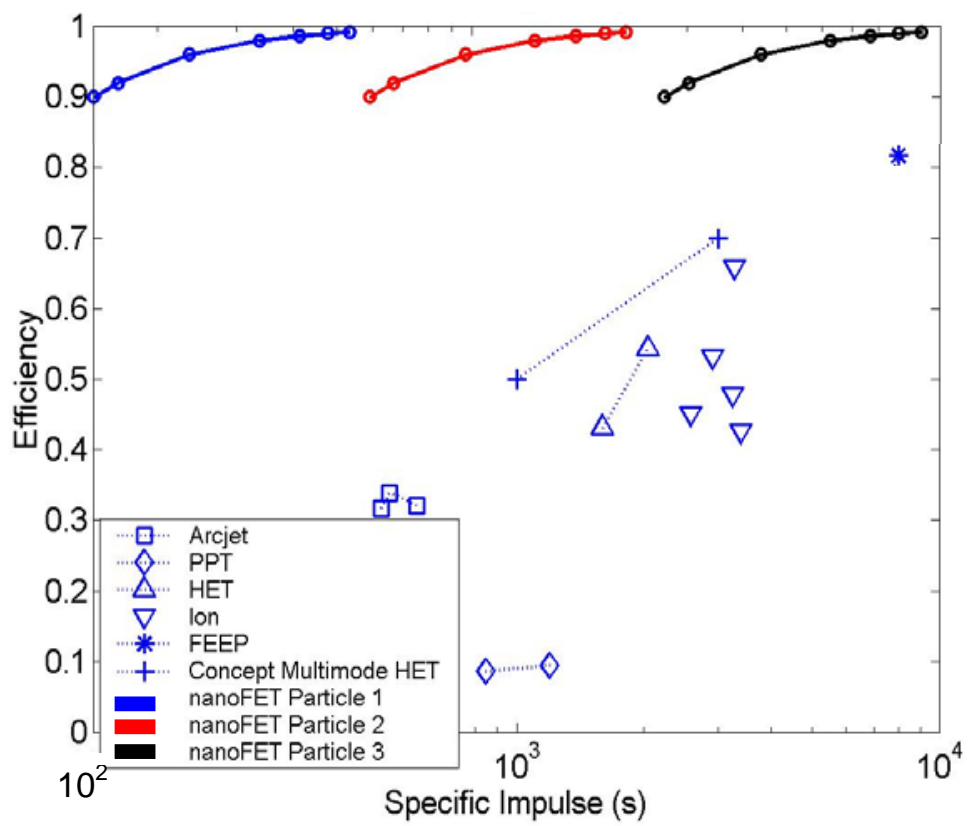

Figure 2. nanoFET's enormous $\boldsymbol{I}_{\mathrm{sp}}$ range at high efficiencies. The accelerating voltages are from $800 \mathrm{~V}$ to $10 \mathrm{kV}$ for carbon nanotubes of (1) 5-nm diameter and 100-nm length, (2) 1-nm diameter and 100-nm length, and (3) 1-nm diameter and 3.5- $\mu$ m length.

This paper focuses on the theoretical aspects of nanoFET operation. Advantages and initial proof-of-concept test results for the nanoFET system are discussed in detail in a parallel paper (AIAA-2006-4803). ${ }^{3}$ 


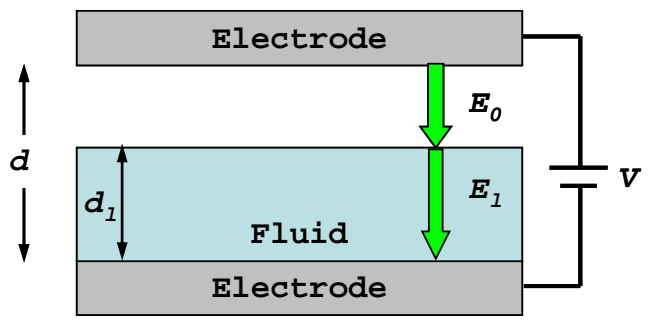

Figure 3. Simplified conceptual model of nanoFET to understand electric fields in the system. A vacuum gap exists between the dielectric liquid and the upper electrode.

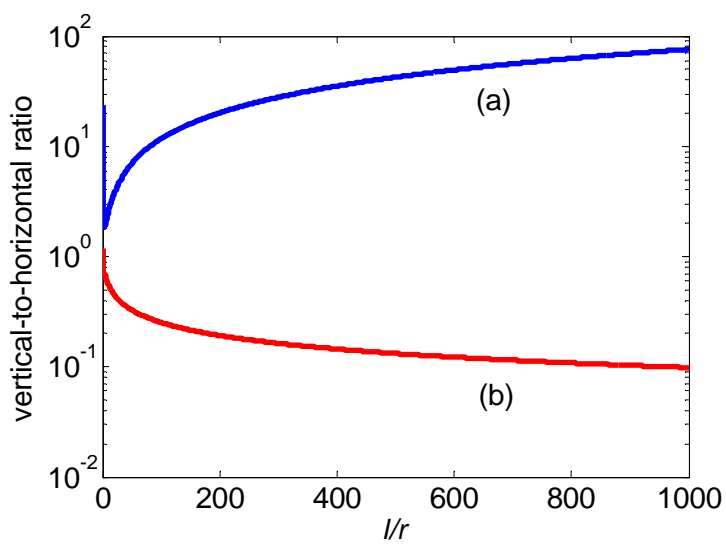

Figure 4. Comparison between vertically and horizontally orientated cylindrical particles. (a) Ratio of acquired particle charge; (b) ratio of required electric field for particle lift-off.

\section{Particle Behavior in nanoFET}

To understand the electric fields and particle behavior during the charging, transport, extraction, and acceleration stages, the nanoFET system can be modeled, as in Figure 3, as a pair of biased electrodes separated by a dielectric liquid layer and a vacuum gap. Within the vacuum gap, the electric field is given by

$$
E_{0}=\frac{V}{d+\left(\frac{\varepsilon_{0}}{\varepsilon_{l}}-1\right) d_{l}}
$$

Within the dielectric liquid, the electric field is

$$
E_{l}=\frac{\varepsilon_{0}}{\varepsilon_{l}} E_{0}=\frac{V}{\frac{\varepsilon_{l}}{\varepsilon_{0}} d+\left(1-\frac{\varepsilon_{l}}{\varepsilon_{0}}\right) d_{l}} .
$$

\section{A. Particle Charging and Lift-Off}

For a charged particle to lift off the electrode, the electrostatic and buoyant forces on it must exceed the gravitational, image charge, and adhesion forces restraining it. In a zero-g space environment, the gravitational and buoyant forces are neglected. For particle sizes on the order of the electrode surface roughness, the adhesion force becomes significant and must be taken into account.

For a conductive spherical particle in contact with an electrode and submersed by the liquid, Félici ${ }^{4}$ gives the acquired particle charge as

$$
q_{0, \mathrm{sp}}=\frac{2 \pi^{3}}{3} r^{2} \varepsilon_{l} E_{l} .
$$

For horizontally orientated cylinders, the acquired charge is

$$
q_{0, \mathrm{cy}-\mathrm{h}}=2 \pi r l \varepsilon_{l} E_{l} .
$$

Proof-of-concept tests have shown that field focusing on a cylindrical particle's tips leads to a moment that rotates the cylinder from a horizontal to a vertical position prior to lift-off. The acquired charge for a vertically orientated cylinder is

$$
q_{0, \mathrm{cy}-\mathrm{v}}=\pi \frac{l^{2}}{\ln \left(\frac{2 l}{r}\right)-1} \varepsilon_{l} E_{l} .
$$

As the cylinder rotates from a horizontal to a vertical orientation, it acquires a larger charge and a greater electrostatic lifting force for a given electric field. Consequently, the minimum required electric field for lift-off decreases as the particle becomes vertical, as seen in Figure 4. 

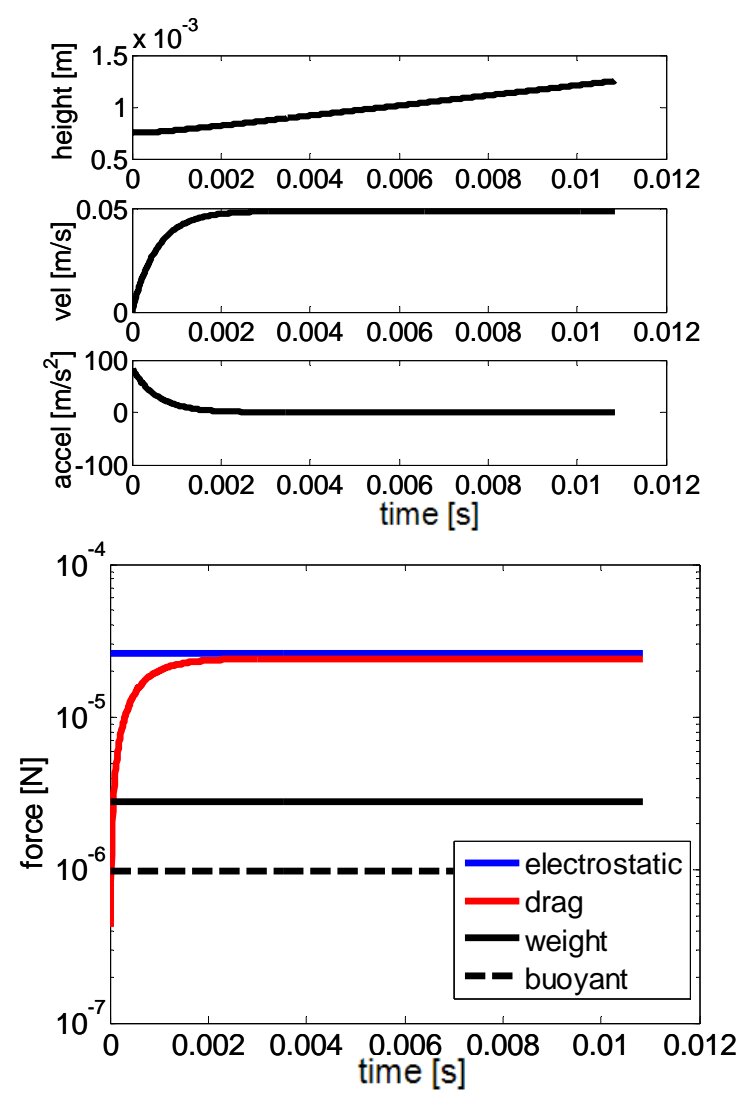

Figure 5. Representative dynamics of particle motion in liquid for proof-of-concept tests. Terminal velocity is quickly reached (top). Electrostatic and drag forces dominate (bottom). Particles are aluminum cylinders, $300 \mu m$ in diameter and $1.5 \mathrm{~mm}$ in length, traversing a 2-mm thick silicone oil layer at $61 \mathrm{~V} / \mathrm{mm}$.

\section{B. Particle Dynamics in Liquid}

Once the particle leaves the electrode surface, the adhesion and electric image forces rapidly vanish, and a fluid drag force now acts to counteract particle motion through the liquid. For the viscous flow regime, the drag force on a sphere is

$$
D=6 \pi \mu_{1} r v
$$

A cylindrical particle can be modeled as an elongated rod moving parallel to its axis. ${ }^{5}$ The fluid drag becomes

$$
D=\frac{2 \pi \mu_{l} l v}{\ln \left(\frac{l}{2 r}\right)+0.193}
$$

As the particle is transported, charge on the conductive particle is gradually leaked to the liquid. The particle charge decreases with time according to

$$
q(t)=q_{0} \exp \left(-\frac{t}{\tau}\right)
$$

with the charge loss time constant given as

$$
\tau \equiv \frac{\varepsilon_{l}}{\sigma_{l}}
$$

The particle's inertial force must account for the "added mass" of the liquid that is accelerated along with the particle. This inertial force is given by

$$
F_{\text {inertial }}=\left(m_{p}+K m_{l}\right) \frac{d v}{d t},
$$

with the coefficient $K$ dependent on particle geometry. For spheres, this coefficient is equal to 0.5. For cylinders modeled as prolate ellipsoids moving parallel to its axis, the "added mass" becomes negligible at high aspect ratios. Assuming that a particle's motion is independent of other particles, the equation of motion for a particle in the liquid is shown below. In zero-g, the buoyant and gravitational forces vanish.

$$
\left(m_{p}+K m_{l}\right) \frac{d v}{d t}=q(t) E_{l}-D+F_{\text {buoyant }}-W .
$$

Figure 5 shows the expected dynamical behavior of the millimeter-sized particles used in the proof-of-concept experiments. A particle quickly reaches terminal velocity in the low-Reynolds number flow and traverses the liquid layer fast enough that charge loss to the liquid is negligible. Figure 5 also indicates the relative magnitudes of the forces acting on a particle while it is in the liquid. Even in the laboratory environment, the electrostatic and drag forces dominate the gravitational forces. As smaller particles are used, the gravitational forces become even less significant. 


\section{Particle Extraction through Liquid Surface}

At the liquid surface, the particle must overcome surface tension forces in order to be extracted. As the particle size becomes smaller, surface tension becomes increasingly significant because it decreases linearly with the scale of the particle while all other forces decrease by a higher power. The surface tension force is given as

$$
F_{\text {surface }}=2 \pi r \gamma \text {. }
$$

Field focusing on the particle leads to an increase in the electrostatic force extracting the particle at the liquid surface. As a first-order estimate, the field enhancement factor is assumed to be

$$
\beta \approx 1+\sqrt{\frac{l}{2 r}},
$$

which is a scaling typically used for carbon nanotube field emitters. ${ }^{6}$ By making simplifying assumptions that the liquid wets the particle completely for the maximum surface tension force and that particle extraction does not take place until a full particle length above the unperturbed liquid surface, the equations of motion can be iterated to determine the particle dynamics during the extraction process. As in the case of particle motion in the liquid, the gravitational forces are negligible compared to the electrostatic, drag, and surface tension forces.

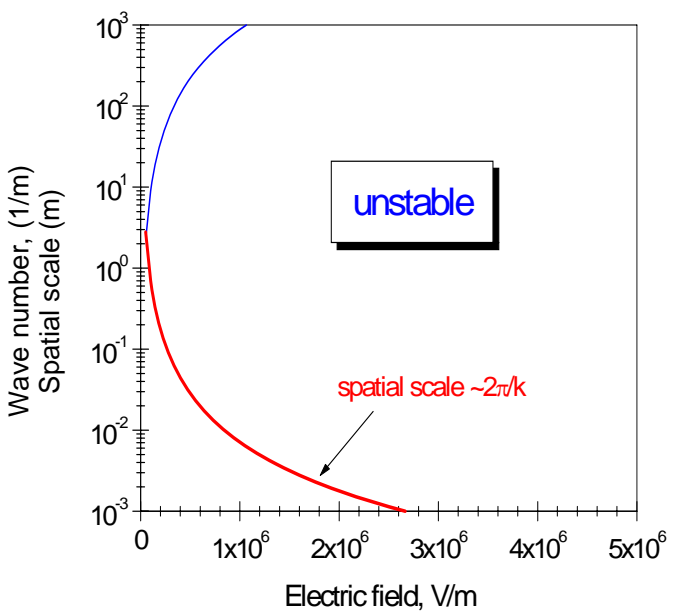

Figure 6. Stability curves for surface electrohydrodynamic waves. Region to the right of the curves represents the regime of wave instability.

\section{Post-Extraction Particle Acceleration}

After extraction from the liquid, the particle emerges in the vacuum gap. Neglecting the insignificant gravitational force, the particle undergoes constant accelerated motion due to the vacuum electric field before being expelled to create thrust.

\section{Liquid Surface Instability}

Under the typical conditions of the colloid thruster, the stable cone jet is considered to be a prerequisite for the generation of droplets. The presence of a high electric field near the liquid surface generally creates surface instabilities that are undesirable for nanoFET because they grow to form Taylor cones that can eject fluid droplets. These colloids, with poorly defined charge-tomass ratios, would reduce the overall nanoFET performance. Fortunately, nanoparticle protrusion from the liquid surface during the extraction process should lead to focusing of the electric field on the particle, thus reducing the likelihood of Taylor cone and liquid jet formation.

At equilibrium in the laboratory setting, the liquid achieves a balance between electric pressure, hydrostatic pressure, and surface tension. Using a corrected version of Tonks's formula ${ }^{7}$ adapted for dielectric liquids, the minimum vacuum electric field that would cause a perturbed liquid surface to become unstable is

$$
E_{0, \text { min }}=\left(\frac{4 g_{0} \gamma \rho_{l}}{\varepsilon_{0}^{2}}\right)^{\frac{1}{4}}\left[1+\left(\frac{\varepsilon_{0}}{\varepsilon_{l}}\right)^{2}\right]^{-\frac{1}{2}} .
$$

As gravitation effects vanish in zero-g, this simple model for the critical electric field must be modified to account for the presence of electrohydrodynamic waves. In general, the dispersion relation for these surface waves in the absence of gravity ${ }^{8}$ is given by 


$$
\omega^{2}=-\frac{\varepsilon_{0} E_{0}^{2}}{\rho_{l}} k^{2}+\frac{\sigma_{l}}{\rho_{l}} k^{3} .
$$

The surface is stable if the wave number satisfies

$$
k>\frac{\varepsilon_{0} E_{0}^{2}}{\sigma_{l}},
$$

or if the characteristic spatial scale of the system is smaller than $1 / k$, as seen in Figure 6 . Therefore, a smaller spatial scale for the system or a smaller electric field would promote surface stability. Further analysis is in progress to establish the main factors affecting liquid surface instability, including flow parallel to the liquid surface as in the nanoFET feed system depicted in Figure 1.

\begin{tabular}{ccc}
\hline \hline $\begin{array}{c}\text { Vacuum } \\
\text { Extraction Electric } \\
\text { Field [V/ } \boldsymbol{\mu m} \text { ] }\end{array}$ & $\begin{array}{c}\text { Al cylinders } \\
\mathbf{( 3 0 0 - \mu \text { dia., }} \\
\mathbf{1 . 5}-\mathbf{m m} \text { length) }\end{array}$ & $\begin{array}{c}\text { Al spheres } \\
\mathbf{( 8 0 0}-\boldsymbol{\mu m} \text { dia.) }\end{array}$ \\
\hline Extraction Model & 1.09 & 1.81 \\
Test Results & $1.32 \pm 0.05$ & $1.78 \pm 0.02$ \\
Model Error & $17 \%$ & $2 \%$ \\
\hline \hline
\end{tabular}

Table 1. Comparison of particle extraction threshold between simplified extraction model and proof-of-concept test results. Results are also displayed in Figure 7.

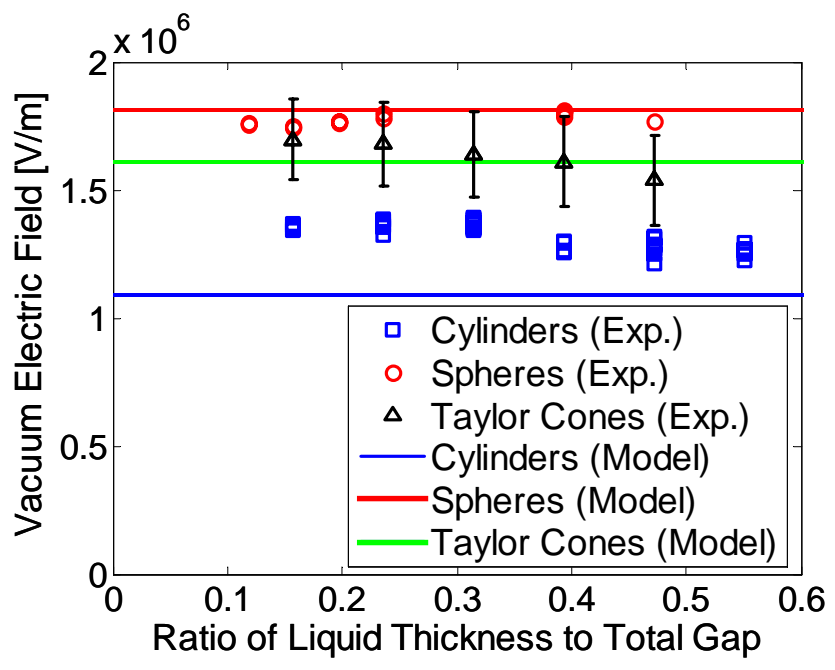

Figure 7. Vacuum electric fields for Taylor cone formation and particle extraction in proof-of-concept tests. Cylindrical (300- $\mu \mathrm{m}$ diameter and 1.5-mm length) and spherical (800- $\mu \mathrm{m}$ diameter) Al particles traversed a total electrode gap of 12.7 $\mathrm{mm}$ partially filled with silicone oil. parallel electrodes and the drag forces associated with the liquid, (2) the fact that the electric field at the charging electrode is not zero, and (3) the realization that charge is actually a function of electric potential since a nanoparticle's charge depends on the electric field. needed to achieve Taylor cone formation and particle extraction in the proof-of-concept tests (see AIAA-2006-4803). For Taylor cone formation, the measured electric fields are in good agreement with Equation 14; for particle extraction, the measured electric fields in Table 1 are within $20 \%$ of the theoretical predictions using the simplified extraction model governed by Equation 13. Note that the extraction threshold for each particle type is essentially independent of liquid thickness because of negligible charge loss to the liquid during the proof-of-concept tests. The proof-of-concept tests have helped to validate the initial theoretical models and have demonstrated that a regime exists where particles may be extracted prior to the onset of liquid surface instability.

\section{Current Density Limitations}

In nanoFET, charge transport through the viscous liquid generates space charge effects that limit the maximum current density. The traditional Child-Langmuir equation describes space charge limited flow of charged particles between two parallel electrodes in a vacuum, assuming that particles start from rest at one electrode and electrostatically accelerate toward the other. ${ }^{9}$ This relationship is given by

$$
j_{\mathrm{CL}}=\frac{4}{9} \varepsilon_{0} \sqrt{\frac{2 q}{m_{p}}} \frac{V^{3 / 2}}{d^{2}} .
$$

Several differences must be taken into account to derive a modified equation applicable for nanoFET. These differences include (1) the

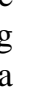

Figure 7 shows the vacuum electric fields

(n)


Consider the case of parallel electrodes infinite in extent in the $x$ - and $y$-directions. From charge conservation, the current density must be constant in the axial direction, or

$$
j=j_{z}=q n v=\text { constant } .
$$

The electric potential must obey Poisson's equation:

$$
\nabla^{2} \varphi=-\frac{q n}{\varepsilon_{l}}
$$

Since the nanoparticles rapidly obtain terminal velocity upon lift-off from the charging electrode, a constant velocity assumption can be made such that the nanoparticle velocity for a given nanoFET configuration depends solely on the local electric field. The particle's terminal velocity in the absence of gravitational forces becomes

$$
v=-\frac{q}{\alpha} \frac{d \varphi}{d z}
$$

where the drag factor (SI units of $\mathrm{kg} / \mathrm{s}$ ) is a function of the particle shape and size as well as the inter-electrode gap and dielectric liquid type:

$$
\alpha \equiv \frac{D}{v}
$$

Poisson's equation thus takes the following form:

$$
\frac{d^{2} \varphi}{d z^{2}}=\frac{j \alpha}{\varepsilon_{l} q \frac{d \varphi}{d z}}
$$

Assuming that the nanoparticle traverses the liquid layer fast enough such that charge loss to the liquid is negligible, multiplying both sides of Equation 22 by $d \varphi / d z$ and integrating results in

$$
\frac{d \varphi}{d z}=\left(\frac{2 j \alpha}{\varepsilon_{l} q} z+E_{z=0}^{2}\right)^{\frac{1}{2}} .
$$

Integrating Equation 23 gives the potential drop across the liquid layer as

$$
V_{l}=\varphi-\varphi_{z=0}=\frac{\varepsilon_{l} q}{3 j \alpha}\left(\frac{2 j \alpha}{\varepsilon_{l} q} z+E_{z=0}^{2}\right)^{\frac{3}{2}}
$$

In the case of a small electric field at the charging electrode's surface compared to the electric field in the electrode gap, the space charge limited current in the viscous liquid is 


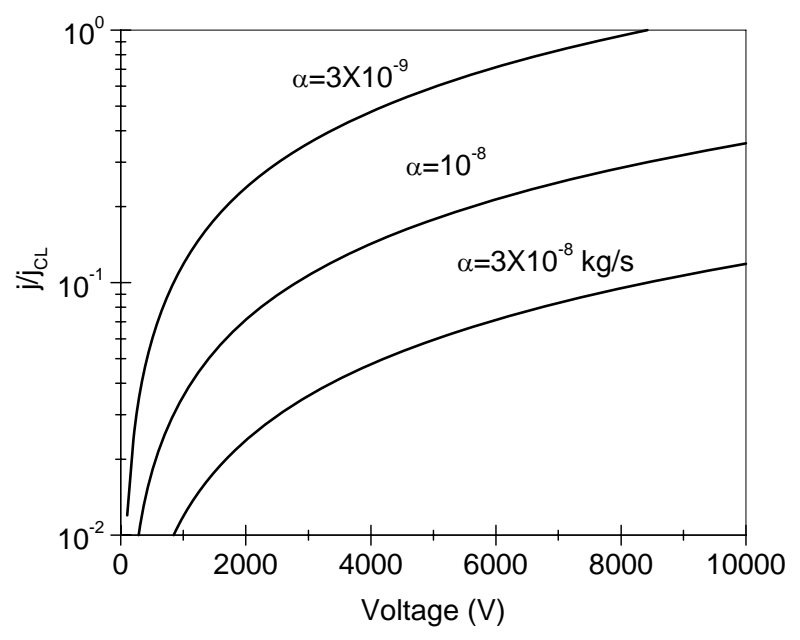

Figure 8. Ratio of current density in viscous flow case to Child-Langmuir current density. Current density decreases with increased drag factor.

$$
j=\frac{9}{8} \varepsilon_{l} \frac{q}{\alpha} \frac{V_{l}^{2}}{d_{l}^{3}} .
$$

The ratio of the current density in the case of viscous media to that described by ChildLangmuir law is plotted in Figure 8 with drag factor as a parameter. Due to drag, the current density decreases in comparison to the vacuum case. Thus, a higher electric field may be required to provide the same current density as in a vacuum case. Breakdown of the dielectric liquid must be avoided in upcoming liquid and inter-electrode gap geometry optimization. In addition, space charge effects due to drag leads to a decrease of the electric field at the charging electrode, thus decreasing the charge delivered to the particle. This effect will be studied further in the future.

\section{Particle Size and Thruster Performance}

In an electric propulsion system, the specific impulse is directly proportional to the square root of particles' charge-to-mass ratio and is given by

$$
I_{\mathrm{sp}}=\frac{1}{g_{0}}\left(2 V_{o} \frac{q}{m_{p}}\right)^{\frac{1}{2}}
$$

The thrust-to-power ratio is inversely proportional to the square root of particles' charge-to-mass ratio and is given by

$$
\frac{T}{P}=\left(\frac{2}{V_{o}} \frac{m_{p}}{q}\right)^{\frac{1}{2}} .
$$

As nanoparticles are scaled down in size for the nanoFET system, their charge-to-mass ratios scale differently depending on the particle shape.

\section{A. Spherical Particles}

The initial particle charge scales with the square of the particle radius. As the particle travels through the liquid, the final particle charge after accounting for charge loss to the liquid can be determined by

$$
\begin{gathered}
q\left(t_{\mathrm{ex}}\right)=q_{0} \exp \left(-\frac{t_{\mathrm{ex}}}{\tau}\right), \\
\propto r^{2} \exp \left(-\frac{1}{r}\right)
\end{gathered}
$$

where the extraction time, neglecting any interaction time with the liquid surface, can be approximated as 


$$
\begin{aligned}
& t_{\mathrm{ex}} \approx \frac{d}{v^{*}} \\
& \propto \frac{1}{r}
\end{aligned} .
$$

Here, the characteristic velocity is set as the particle's terminal velocity, which is quickly reached, in the absence of gravitational forces and charge loss with the liquid:

$$
\begin{aligned}
v^{*} & \equiv \frac{\pi^{2}}{9} \frac{\varepsilon_{l} E_{l}^{2}}{\mu_{l}} r . \\
& \propto r
\end{aligned}
$$

The final charge-to-mass ratio scales as

$$
\frac{q\left(t_{\mathrm{ex}}\right)}{m_{p}} \propto \frac{1}{r} \exp \left(-\frac{1}{r}\right),
$$

which decreases with smaller particle sizes. Thus, spherical particles of decreasing radii improve the thrust-to-power ratio but decrease the $I_{\mathrm{sp}}$ for a given emitter configuration and accelerating potential. For a fixed emitter geometry, the required electric field to extract particles from the liquid surface increases with decreased particle size. As the particle size decreases, so does its terminal velocity in the liquid. Since the particle now takes longer to traverse the liquid layer, it loses more charge to the surrounding liquid before reaching the surface. Consequently, a larger electric field is needed to extract the particle of reduced charge. To overcome this problem, the liquid thickness must be scaled along with the particle size to reduce the liquid layer transit time.

\section{B. Cylindrical Particles}

A similar analysis with cylindrical particles follows. As the particle travels through the liquid, the final particle charge after accounting for charge loss to the liquid can be determined by

$$
\begin{gathered}
q\left(t_{\mathrm{ex}}\right)=q_{0} \exp \left(-\frac{t_{\mathrm{ex}}}{\tau}\right) \\
\propto \frac{l^{2}}{\ln (4 A)} \exp \left[-\frac{\ln (4 A)}{\ln A}\right]
\end{gathered}
$$

where the aspect ratio is large and given as

$$
A=\frac{l}{2 r}>>1 .
$$

Setting the characteristic velocity as the particle terminal velocity in the absence of gravitational forces and charge loss with the liquid, 


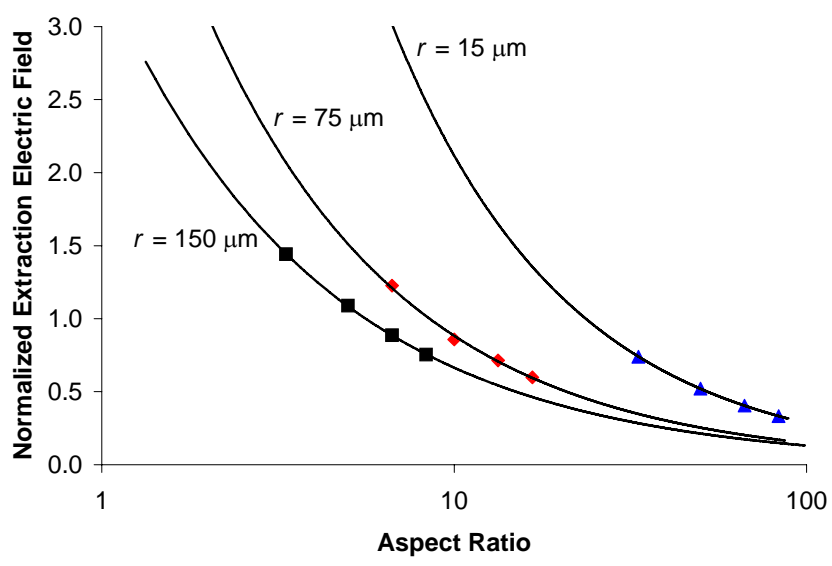

Figure 9. Vacuum extraction field simulations with small cylindrical particles. The required extraction field decreases with larger particle radii and larger aspect ratios.

$$
\begin{aligned}
v^{*} & \equiv \frac{1}{2} \frac{\varepsilon_{l} E_{l}^{2}}{\mu_{l}} \frac{l \ln A}{\ln (4 A)}, \\
& \propto \frac{l \ln A}{\ln (4 A)},
\end{aligned}
$$

the final charge-to-mass ratio scales as

$$
\frac{q\left(t_{\mathrm{ex}}\right)}{m_{p}} \propto \frac{A^{2}}{l \ln (4 A)} \exp \left[-\frac{\ln (4 A)}{l \ln A}\right],
$$

which decreases with shorter cylinders but increases with longer aspect ratios. For a given emitter configuration and accelerating potential, a higher $I_{\mathrm{sp}}$ can be achieved by increasing the cylindrical particle aspect ratio. As in the case of the spherical particles, liquid thickness should be scaled down along with the particle length to reduce charge loss to the liquid during particle transport to the surface. Results from numerical simulations based on small cylindrical particles for the proof-of-concept tests are shown in Figure 9.

\section{Thruster Performance}

By adjusting the nanoparticle size, an enormous range of $I_{\mathrm{sp}}$ may be achieved at high efficiencies. Figure 2 shows the nanoFET system potentially spanning an $I_{\mathrm{sp}}$ range of 100 to $10,000 \mathrm{~s}$ at greater than $90 \%$ thrust efficiency with three types of carbon nanotube particles. Inefficiencies in the nanoFET system are due to (1) viscous drag in the liquid, (2) charge loss to the liquid, (3) particle impingement on the MEMS gates, and (4) beam defocusing. The last two sources of inefficiencies are dependent on the MEMS gate design, ${ }^{10}$ but the impact of the first two sources of inefficiencies may be lessened by reducing the liquid thickness.

\section{Conclusions \& Future Work}

Preliminary models for particle charging, transport, and extraction along with liquid surface instability have been developed for the nanoFET system. These models have been validated by initial proof-of-concept tests for submillimeter-sized particles and are in the process of being extended to the nanoparticle scale. Work is currently continuing on refining the understanding of the electrode adhesion force for nanoparticles, liquid surface instability in zero-g, and the physics of particle extraction through a liquid surface. Optimization of nanoFET's performance based on geometric configurations as well as particle and liquid properties are also on-going.

\section{Acknowledgments}

This project is funded by a Phase 1 grant (NAS5-03110/07605-003-051) from the NASA Institute for Advanced Concepts and supported by a National Defense Science and Engineering Graduate Fellowship. Thanks to Matthew Forsyth at the University of Michigan for helping to construct the experimental setup being used to validate the initial theoretical models.

\section{References}

\footnotetext{
${ }^{1}$ Musinski, L., Liu, T., Gilchrist, B., Gallimore, A., and Keidar, M., "Scalable Flat-Panel Nano-Particle MEMS/NEMS Thruster,” IEPC-2005-176, 29th International Electric Propulsion Conference, Princeton, NJ, 31 October - 4 November 2005.

${ }^{2}$ Chesta, E., Nicolini, D., Robertson, D., and Saccoccia, G., "Experimental Studies Related to Field Emission Thruster Operation: Emission Impact on Solar Cell Performances and Neutralization Electron Backstreaming Phenomena,” IEPC-2003102, 28th International Electric Propulsion Conference, Toulouse, France, 17-20 March 2003.

${ }^{3}$ Musinski, L., Liu, T., Gilchrist, B., Gallimore, A., and Keidar, M., "Nanoparticle Electric Propulsion: Experimental Results,” AIAA-2006-4803, 42nd Joint Propulsion Conference, Sacramento, CA, 9-12 July 2006.
} 
${ }^{4}$ Félici, N.-J., "Forces et charges de petits objets en contact avec une électrode affectée d’un champ électrique,” Revue Générale de l'Électricité, 75 (1966), pp. 1145-60.

${ }^{5}$ Tobazéon, R., "Electrohydrodynamic behaviour of single spherical or cylindrical conducting particles in an insulating liquid subjected to a uniform DC field,” J. Phys. D: Appl. Phys., 29 (1996), pp. 2595-608.

${ }^{6}$ Smith, R.C., Carey, J.D., Forrest, R.D., Silva, S.R.P., "Effect of aspect ratio and anode location on the field emission properties of a single tip based emitter," J. Vac. Sci. Technol. B 23(2), Mar/Apr 2005, pp. 632-5.

7 Tonks, L., “A Theory of Liquid Surface Rupture by a Uniform Electric Field,” Physical Review, Vol. 48, 15 September 1935, pp. 562-8.

${ }^{8}$ Suvorov, V. and Zubarev, N., "Formation of the Taylor cone on the surface of liquid metal in the presence of an electric field,” J. Phys. D: Appl. Phys., 37 (2004), pp. 289-97.

${ }^{9}$ Child, C., Phys. Rev., 32 (1911), p. 492; Langmuir, I., ibid, 21 (1921), p. 419.

${ }^{10}$ Liu, T., Morris, D., Cionca, C., Gallimore, A., Gilchrist, B., and Clarke, R., "MEMS Gate Structures for Electric Propulsion Applications,” AIAA-2006-5011, 42nd Joint Propulsion Conference, Sacramento, CA, 9-12 July 2006. 